Measurement of mortar permittivity during setting using a coplanar waveguide

This article has been downloaded from IOPscience. Please scroll down to see the full text article.

2010 Meas. Sci. Technol. 21045702

(http://iopscience.iop.org/0957-0233/21/4/045702)

View the table of contents for this issue, or go to the journal homepage for more

Download details:

IP Address: 147.83.10.124

The article was downloaded on 13/05/2011 at 13:37

Please note that terms and conditions apply. 


\title{
Measurement of mortar permittivity during setting using a coplanar waveguide
}

\author{
P Juan-García and J M Torrents \\ Department of Electronic Engineering, Technical University of Catalonia, Jordi Girona 1-3, Building C4, \\ 08034 Barcelona, Spain \\ E-mail: torrents@eel.upc.edu
}

Received 11 November 2009, in final form 2 February 2010

Published 9 March 2010

Online at stacks.iop.org/MST/21/045702

\begin{abstract}
A sensor based on a coplanar waveguide structure was designed to perform non-destructive tests for material characterization in which the measurement can be done only on one side of the sample. The measurements were compared with the impedance of a capacitor filled with the same material. The permittivity and insertion loss of the sensor showed valuable information about the setting process of a mortar slab during the first 28 days of the hardening process, and a good correlation between both measurements was obtained, so the proposed setup can be useful for structural surveillance and moisture detection in civil structures.
\end{abstract}

Keywords: coplanar waveguide, mortar non-destructive testing, permittivity

\section{Introduction}

The electromagnetic characterization of construction materials, such as concrete or mortar, gives the chance to monitor structures. Nowadays, the use of non-destructive testing applied to civil structures is gaining significance, and many authors have recently developed different methods to estimate some physical parameters from an electromagnetic measurement.

Previous works have reported good results working with low frequency (LF) impedance measurements (Torrents et al 2001), but showed great difficulties when applying them in a field context. Some of these problems can be reduced by using microwaves, due to their capability to penetrate through solid materials, so in this work frequencies up to $3 \mathrm{GHz}$ were used. Using a time domain reflection (TDR) technique, $\mathrm{Xu}$ et al (2002) and other authors have developed embedded sensors to monitor the evolution of electromagnetic constants during setting (mainly permittivity and conductivity), and particularly the monitoring of moisture in concrete has been studied using coaxial sensors (Sokoll and Jacob 2007). Huebner and Kupfer (2007) developed a planar TDR sensor, to be surrounded by the material under test. However, there is a need to develop methods to measure big structures or precast walls, so embedding sensors are sometimes not easily put into practice. Many non-contact sensors have been studied which can be placed in contact with the sample under test in order to provide useful information about its physical properties (Volgyi 2007, Yoo Jin et al 2003).

Similarly, the use of transmission lines for the characterization of concrete reported good results, using time domain transmission (TDT) or frequency domain transmission (FDT) procedures. In these experiments, the sensor, both a waveguide (Pauli et al 2007) and a coaxial line (Millard et al 2001), was filled with concrete and the propagation delay gave information about the dielectric constant and loss factor of the material. Nonetheless, these tests are of application only in a laboratory situation but not for field structures. Based on the same principle, Stuchly and Bassey (1998) designed a surface sensor attached to the sample under test to perform dielectric measurements.

The measurement of mortar setting has been explored using similar methods, showing a permittivity decrease as the sample hardens (Roqueta et al 2009). If the dielectric constant is unchanging for a certain range of frequencies, an effective permittivity is proportional to the phase slope of the transmitted wave.

The aim of this work is to measure the evolution of the dielectric constant and loss factor of a mortar sample during the setting process by a surface sensor, a promising sensing system to give reliable information about mortar setting. This is a laboratory experience. The long-term goal of this system is to apply this experience to the surveillance of field structures. 


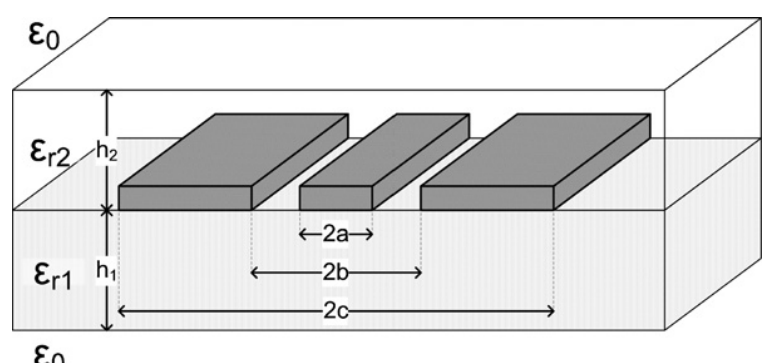

Figure 1. Geometrical model of a two-layer coplanar waveguide.

\section{Principle of measurement}

A TDT/FDT technique was applied to a sensor with two ports attached to one of the faces of the sample in which the analysis of the $s_{21}$ parameter was provided with useful information about the setting process of the mortar tested. The wave was transmitted through a coplanar waveguide (CPW), a common structure based on three conductors on the surface of a substrate (Wen 1969). The material permittivity is obtained numerically from the theoretical model of a multilayer coplanar waveguide with finite-width ground planes (Simons 2001, chapter 4). The signal attenuation provides information about the loss factor, which is directly related to the water content (Juan-García and Torrents 2009).

The measured permittivity is a combination of the dielectric constant of the mortar, the substrate and the surrounding air. Therefore, an effective permittivity $\left(\varepsilon_{\text {eff }}\right)$ is defined in the design equations. As the dielectric constant of the substrate and air are perfectly known, from the measurement of the effective permittivity of the whole setup, the real permittivity can be numerically estimated. The characteristic impedance can be calculated as

$$
Z_{0}=\frac{30 \pi}{\sqrt{\varepsilon_{\mathrm{eff}}}} \frac{K(k)}{K\left(k^{\prime}\right)}(\Omega),
$$

where $K(x)$ is the complete elliptic integral of the first kind, and

$$
k=\frac{c}{b} \sqrt{\frac{b^{2}-a^{2}}{c^{2}-a^{2}}}=\sqrt{1-k^{\prime 2}},
$$

where $a, b$ and $c$ are geometrical dimensions of the CPW as shown in figure 1.

In this work, a two-layer CPW was designed, so the effective permittivity was calculated using formulae developed for multilayered CPW with finite ground planes (Simons 2001, chapter 4):

$$
\varepsilon_{\mathrm{eff}}=1+\sum_{i=1}^{2} \frac{1}{2}\left(\varepsilon_{r_{i}}-1\right) \frac{K(k)}{K\left(k^{\prime}\right)} \frac{K\left(k_{i}^{\prime}\right)}{K\left(k_{i}\right)},
$$

where

$$
k_{i}=\frac{\sinh \left(\frac{\pi c}{2 h_{i}}\right)}{\sinh \left(\frac{\pi b}{2 h_{i}}\right)} \sqrt{\frac{\sinh ^{2}\left(\frac{\pi b}{2 h_{i}}\right)-\sinh ^{2}\left(\frac{\pi a}{2 h_{i}}\right)}{\sinh ^{2}\left(\frac{\pi c}{2 h_{i}}\right)-\sinh ^{2}\left(\frac{\pi a}{2 h_{i}}\right)},}
$$

where $h_{1}$ and $h_{2}$ are geometrical dimensions of the CPW, and $\varepsilon_{r i}$ is the dielectric constant of each layer, as shown in figure 1 .
The propagation of an electromagnetic wave along a transmission line is defined by its propagation constant. For a non-magnetic material, it is given by

$$
\gamma=\alpha+\mathrm{j} \beta=\mathrm{j} \frac{2 \pi}{\lambda}\left(\varepsilon^{\prime}-\mathrm{j} \varepsilon^{\prime \prime}\right)^{1 / 2}
$$

where $\lambda$ is the free space wavelength, $\alpha$ is the attenuation constant, $\beta$ is the phase constant, $\varepsilon^{\prime}$ is the dielectric constant and $\varepsilon^{\prime \prime}$ is the loss factor (von Hippel 1954, chapter 1). The dielectric constant and loss factor can be expressed as

$$
\varepsilon^{\prime}=\frac{\lambda^{2}}{4 \pi^{2}}\left(\beta^{2}-\alpha^{2}\right) \quad \varepsilon^{\prime \prime}=\frac{\lambda^{2}}{2 \pi^{2}} \alpha \beta
$$

For low-loss materials $\left(\varepsilon^{\prime \prime} / \varepsilon^{\prime}<0.3\right)$, the propagation parameters can be simplified (Stuchly and Bassey 1998) as

$$
\alpha \approx \frac{\pi}{\lambda} \frac{\varepsilon^{\prime \prime}}{\sqrt{\varepsilon^{\prime}}} \quad \beta \approx \frac{2 \pi}{\lambda} \sqrt{\varepsilon^{\prime}} .
$$

\section{Material and methods}

\subsection{Sample preparation}

Two identical mortar samples were prepared using ordinary portland cement and calcareous sand, passed through a $5 \mathrm{~mm}$ sieve, with a water/cement ratio of 0.50 and a sand/cement ratio of 2, without using additives. The samples were cast on $350 \times 180 \times 40 \mathrm{~mm}$ plastic moulds. A surface sensor was attached to one of the moulds, and the mortar was poured over it. A capacitor was embedded in the other sample to monitor permittivity changes by impedance measurements, as is detailed below. The permittivity measurement using a capacitor was performed to compare the results with the CPW measurement.

\subsection{Coplanar sensor design}

A $300 \times 150 \times 1.6 \mathrm{~mm}^{3}$ coplanar waveguide with finite-width ground planes was designed in order to measure the dielectric constant from only one face of the sample under test, using the equations presented in section 2. The CPW, fabricated on a FR4 substrate, has a central conductor of $3 \mathrm{~mm}$ and a maximum gap of $62 \mathrm{~mm}$. It was adapted to $50 \Omega$ in the input port and widened to reach an impedance of $200 \Omega$ in its broader end, using a 1:4 exponential impedance transformer to minimize the reflected wave. The length of the taper $(15 \mathrm{~cm})$ is directly related to the low-frequency limit (Pozar 2005, chapter 5), in this case fixed at $300 \mathrm{MHz}$, and the dimensions of the taper were based on an ideal model of the mortar as an homogeneous material with a permittivity of 5 . The maximum penetration of the wave inside the material is theoretically limited by $75 \%$ of the gap between the conductors (Seagar et al 1987), so the dimensions of the line were designed in accordance with the sample thickness. 


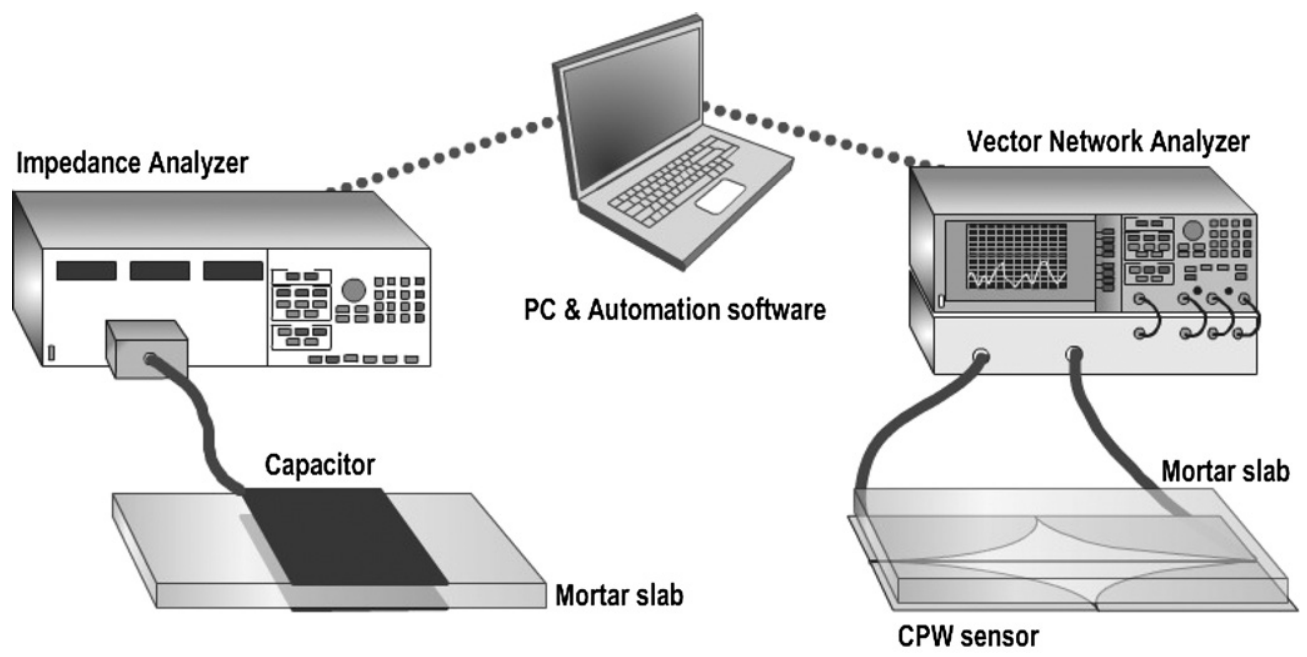

Figure 2. Diagram of the measurement setup.

\subsection{Capacitor design}

Two $150 \times 150 \mathrm{~mm}^{2}$ copper sheets separated $35 \mathrm{~mm}$ from each other were embedded in one of the mortar samples, creating a capacitor between them. Once the mortar was poured, the empty space between them was completely filled with the material under test, so the measurement of capacitance variations showed changes in the material permittivity that were correlated afterwards with the data obtained from the coplanar sensor.

According to Elliott (1966, p 189), the capacitance of this structure $(a=0.15 \mathrm{~m}, h=0.035 \mathrm{~m})$ can be expressed as

$$
\frac{C}{\varepsilon_{\text {eff }}}=\frac{a^{2}}{h}\left[1+\frac{2 h}{\pi a} \ln \left(\pi \frac{a}{h}\right)\right] .
$$

\subsection{Measurement instruments}

Reflection and transmission parameters of the coplanar sensor were obtained with an HP8753C vector network analyser (VNA) by performing a wideband frequency sweep from $300 \mathrm{MHz}$ to $3 \mathrm{GHz}$. 401 points per sample were measured, leading to a resolution of $6.73 \mathrm{MHz}$, with an input power of $0 \mathrm{dBm}$. The measurements were then calibrated using a TRL algorithm (Engen and Hoer 1979), more suitable for planar structures than the traditional SOLT.

The capacitance measurements were acquired using an LF impedance analyser, HP4192A, measuring ten points per decade from $10 \mathrm{~Hz}$ to $10 \mathrm{MHz}$ with $1.1 \mathrm{~V}_{\mathrm{RMS}}$ voltage excitation. All the data were acquired by a PC via GPIB connection, using a LabView automation driver, sampling each 5 min for 28 days. A schematic diagram of the whole setup is provided in figure 2 . The temperature inside the sample and in the environment was measured with a thermocouple using a Fluke 185 multimeter as a data logger.

\section{Results and discussion}

The coplanar sensor was placed in the mould with the copper sheet in the upper part before pouring the mortar over it (figure 3). Previous works showed the setting process of mortar by putting the sensor over the mortar (Juan-García and Torrents 2009), but since the surface of the mortar hardens in the first stages, no significant changes were observed after the first $24 \mathrm{~h}$. However, by placing the sensor in the inner part of the mould, relevant changes in the permittivity can be observed during the whole setting process. Moreover, the gap between the sensor and the sample must be perfectly known in order to estimate the material permittivity from the measurement of the effective permittivity, so by pouring the mortar over the sensor a lack of gaps is ensured, whilst when measuring in the surface, air bubbles would appear, changing the effective permittivity and its effect should be taken into account.

Once the phase slope is processed, an effective permittivity value is obtained for each time sample. The mortar permittivity was estimated afterwards by numerical computation using a model of the multilayer CPW with finitewidth ground planes described in more depth in Simons (2001, chapter 4), using a three-layer model analogous to the model presented in section 2 (adding a third layer of polyethylene), taking into account the permittivity of the surrounding air $\left(\varepsilon_{r}=1.0\right)$, the FR4 substrate $\left(\varepsilon_{r}=4.2\right)$ and the mould, made of polyethylene $\left(\varepsilon_{r}=2.3\right)$. The estimation was performed applying the analytical model of a single $\mathrm{CPW}$ with finite ground planes to the tapered line. The line was divided into 300 segments (each one being $1 \mathrm{~mm}$ long), as shown in figure 4. Each segment has its own propagation velocity, which is related to the effective permittivity of the coplanar waveguide for each geometry, so the theoretical impedance was calculated for each segment of the tapered coplanar waveguide and the obtained values were finally averaged.

It has to be stated that in the initial stages, there was a high amount of water in the mixture, so high losses were expected, because the effects of conductivity are far more relevant than those of permittivity. What is more, there is a certain range of frequencies in which the amplitude of the transmitted signal was under the sensitivity of the measurement instrument, so a cut-off effect appears. The upper limit of suitable frequencies for this measurement (figure 5) starts around $1.4 \mathrm{GHz}$, so the 


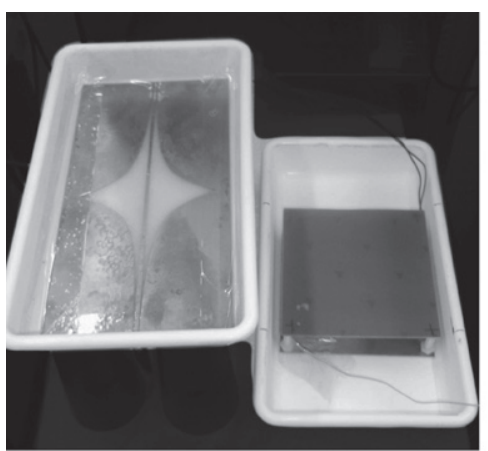

(a)

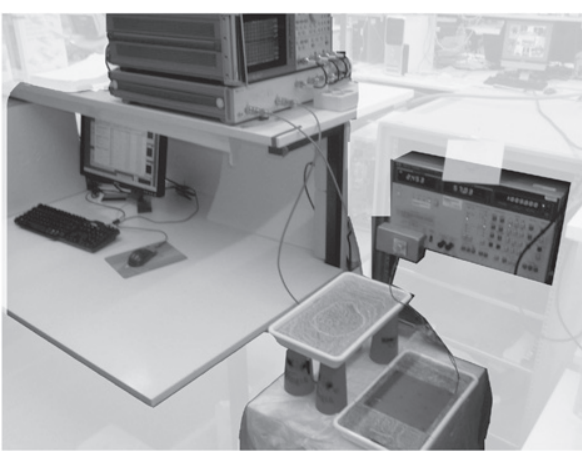

(b)

Figure 3. Setup (a) before pouring the mortar and $(b)$ after pouring the mortar.

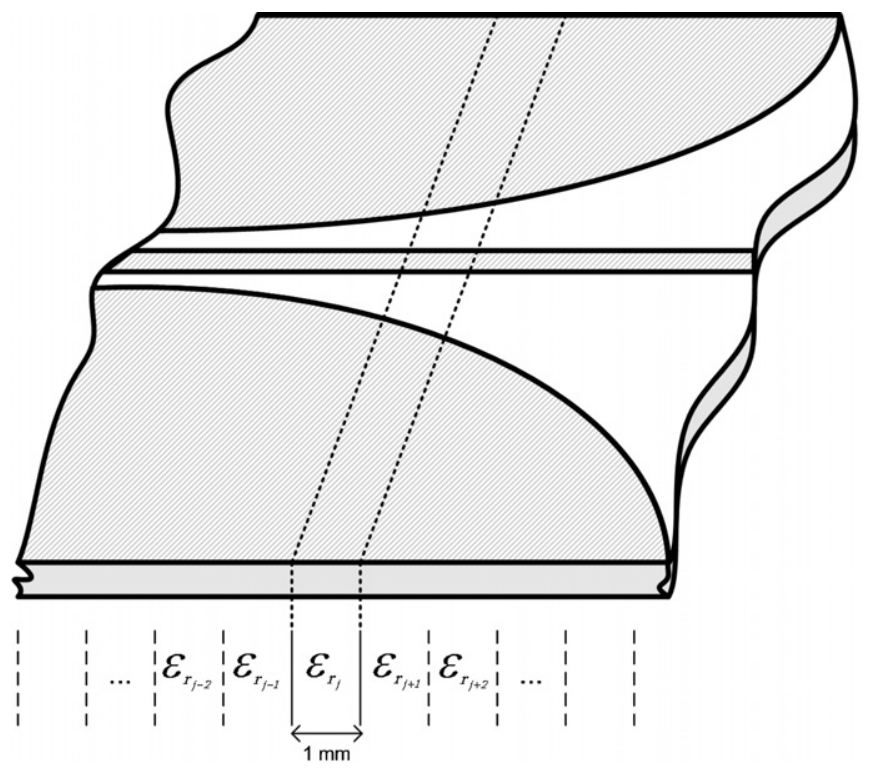

Figure 4. Diagram for the calculation of the effective permittivity as a function of the geometry of the coplanar waveguide.

results of both the permittivity and the insertion loss were obtained under $1 \mathrm{GHz}$.

The initial permittivity is around 20, mainly due to the great amount of water $\left(\varepsilon_{r}=80\right)$ in the sample. As the sample sets and the hydration process takes place, the amount of free water diminishes, so the dielectric constant decreases proportionally (see figure 6). As was expected, the main variations occur in the first $24 \mathrm{~h}$, when the sample loses its viscosity and hardens significantly. The permittivity values for set mortar, which were obtained using equation (7), are in accordance with the measurements performed by other authors using different methods (Peer et al 2003). However, assuming that (7) is valid when $\varepsilon^{\prime \prime} / \varepsilon^{\prime}<0.3$, the permittivity measurements using the CPW method are significant after the first $18 \mathrm{~h}$. From the beginning of the experiment up to $18 \mathrm{~h}$, the high values of conductivity make this assumption wrong and distort the permittivity results using the approximation in (7).

As for the insertion loss, it shows a similar trend to the permittivity, but is frequency dependent. The initial amount of water leads to a value of nearly $60 \mathrm{~dB}$ (figure 6) and rapidly

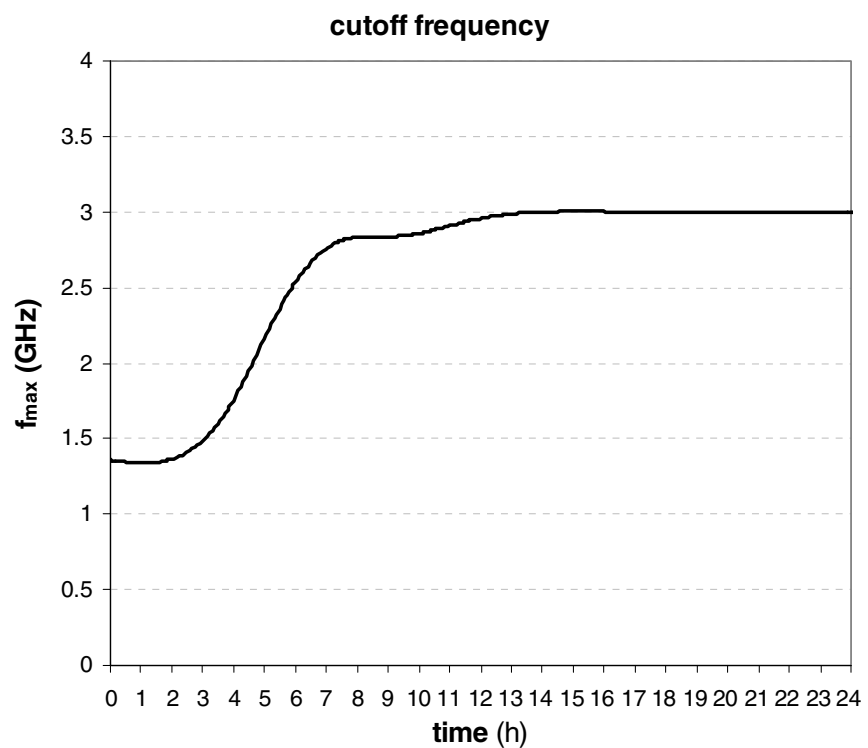

Figure 5. Maximum suitable frequency against time.

decreases while the free water reacts with the cement forming a conductive matrix filled with dielectric gaps. Similar to the permittivity behaviour, the loss factor changes significantly for weeks, although the main changes occur in the first $24 \mathrm{~h}$.

To validate the results, both the permittivity and loss measurements were compared with the measurements performed with the capacitor at low frequencies. However, the permittivity measured with the capacitor system showed values over 1000. According to the literature (Macdonald and Barsoukov 2005, p 469), the double-layer effect is the cause of those high values of permittivity. The capacitor measurements are performed at low frequency $(100 \mathrm{kHz})$, which entails a high ion exchange phenomenon, whereas the CPW measurements are performed over $300 \mathrm{MHz}$, so the ion exchange is far lower. Thus, the capacitive measurement is highly affected by the double-layer effect, whilst the CPW measurement leads to more accurate permittivity results. Despite the fact that the absolute values obtained were different with the capacitor and the CPW line, both values were normalized with respect to the average value over the time. Such comparison showed good agreement in their time changes (figure 7), except in the initial stages, due to the high conductivity of water, whereas 


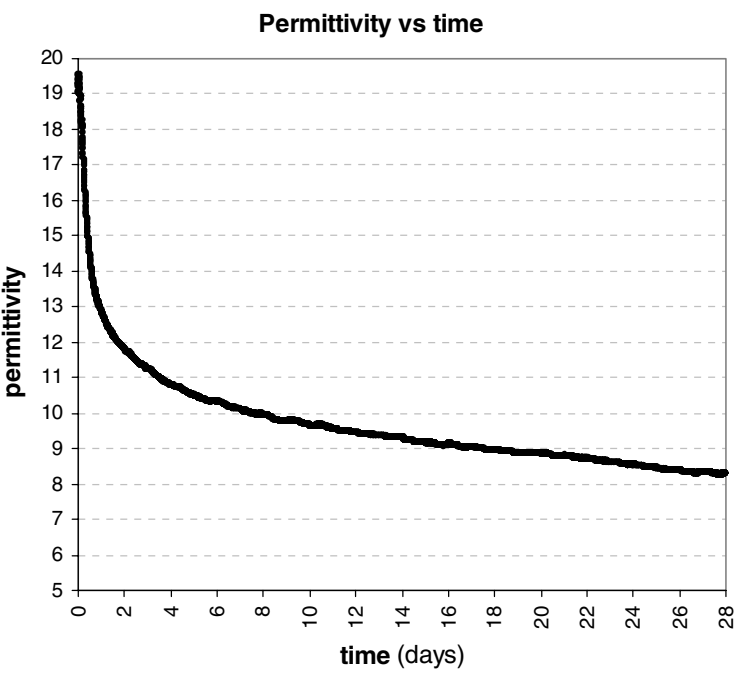

(a)

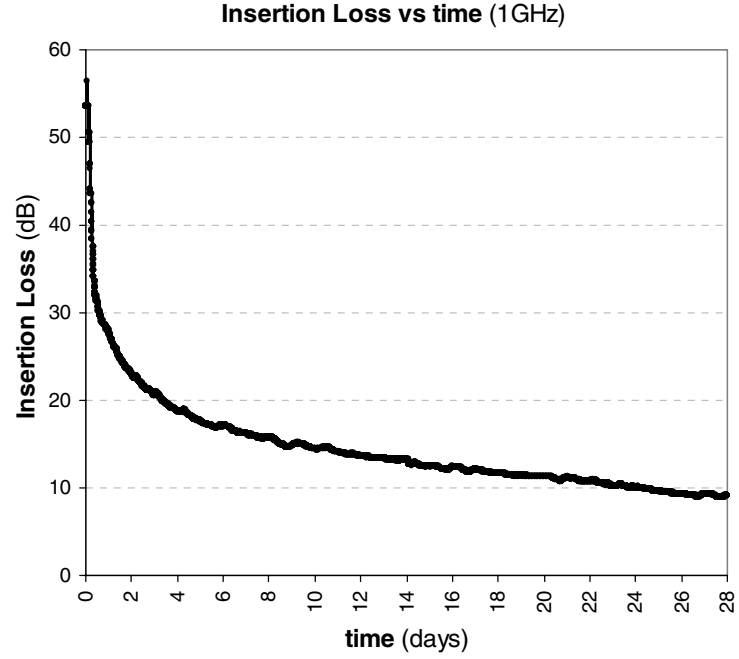

(b)

Figure 6. Setting progress results: (a) permittivity measured from $300 \mathrm{MHz}$ to $1 \mathrm{GHz}$ and $(b)$ insertion loss measured at $1 \mathrm{GHz}$.

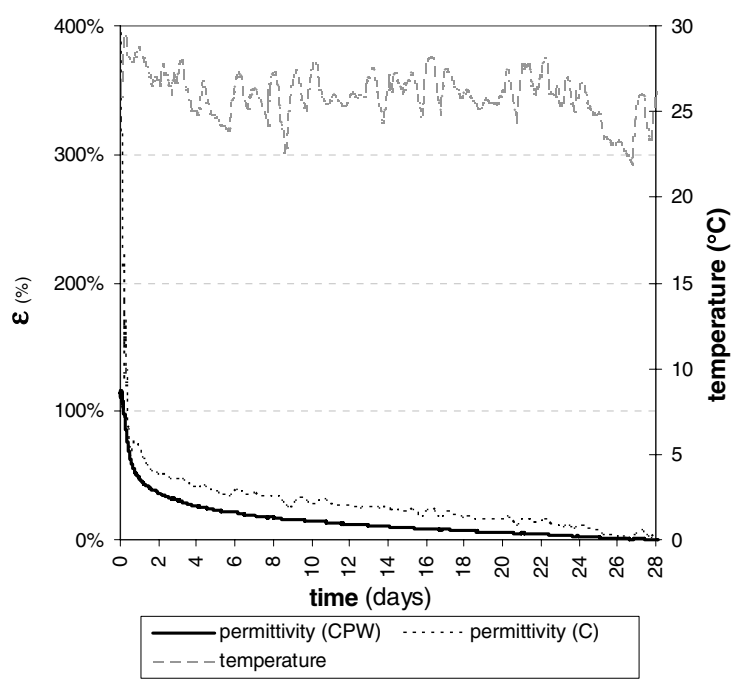

(a)

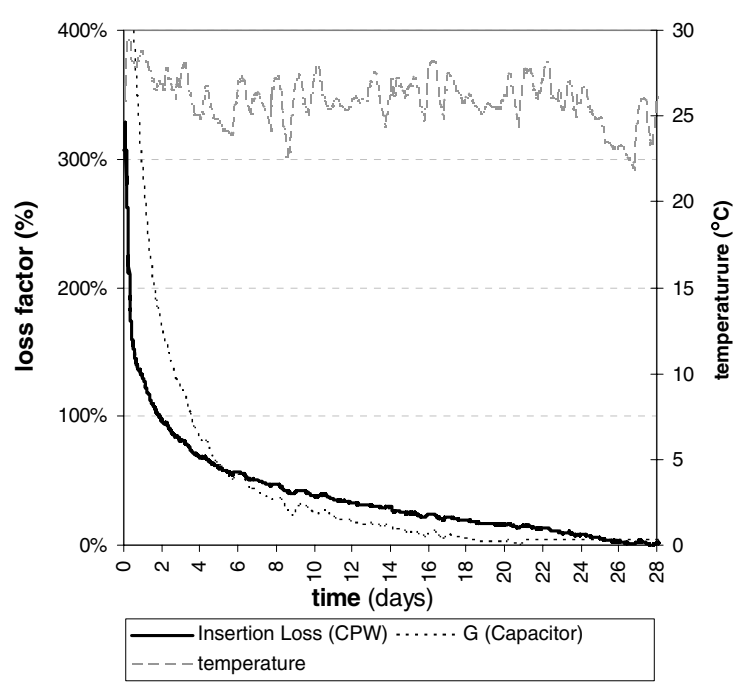

(b)

Figure 7. (a) Permittivity progress measured by the CPW sensor (from 0.3 to $1 \mathrm{GHz}$ ) and the capacitor (at $100 \mathrm{kHz}$ ). (b) Insertion loss (at $1 \mathrm{GHz}$ ) and conductance of the capacitor measurement (at $100 \mathrm{kHz}$ ). Temperature measurements are included to show the thermal dependence of the capacitor (LF) measurements.

the CPW measured feasible values (with a loss of resolution because of the high attenuation of the signal).

Moreover, temperature changes led to variations in the permittivity measured with the capacitor (as well as the insertion loss), but the measurement of permittivity using a CPW was hardly affected by temperature variations.

\section{Conclusions}

The setting progress of a mortar sample was measured for 28 days using a new coplanar sensor in the range of microwaves. The dielectric constant was measured, as well as the insertion loss factor, and good results were obtained, which showed a decreasing trend of the permittivity as the sample hydrated, and a decreasing insertion loss associated too with the amount of water in the sample. From the measurement of the transmission parameters of the transmission line, and using its theoretical model, the permittivity of the material was estimated. The measurements showed good agreement with the impedance at low frequencies of a capacitor filled with the same material, but were more reliable using the CPW sensor due to the high amount of water in the sample, particularly in the early stages. The suitable frequencies for the permittivity measurements were studied, and a maximum cut-off frequency was defined. The promising results of this experiment open the door to further works in a field context.

\section{Acknowledgments}

This work was partially supported by the Ministerio de Educación y Ciencia under project BIA 2005-09412-C03-03 and by the FCC company, as part of the HATCONS project. 
The authors acknowledge Professor Antonio Aguado for his warm advice about sensing and samples preparation.

\section{References}

Elliott R S 1966 Electromagnetics (New York: McGraw-Hill)

Engen G F and Hoer C A 1979 Thru-reflect-line: an improved technique for calibrating the dual six-port automatic network analyzer IEEE Trans. Microw. Theory Tech. 27 987-93

Huebner C and Kupfer K 2007 Modelling of electromagnetic wave propagation along transmission lines in inhomogeneous media Meas. Sci. Technol. 18 1147-54

Juan-García P and Torrents J M 2009 Measurement of moisture in mortar using a coplanar waveguide XIX Imeko World Congress. Fundamental and Applied Metrology (Lisboa, Portugal) pp 2285-8

Macdonald J R and Barsoukov E 2005 Impedance Spectroscopy: Theory, Experiment, and Applications (Hoboken, NJ: Wiley-Interscience)

Millard S G, Al-Qadi I L, Shaw M R, Riad S M, Sharri A and Bungey J H 2001 Coaxial transmission lines: development of test procedures for concrete J. Mater. Civil Eng. 13 202-8

Pauli M, Kayser T and Wiesbeck W 2007 A versatile measurement system for the determination of dielectric parameters of various materials Meas. Sci. Technol. 181046

Peer S, Case J T, Gallaher E, Kurtis K E and Zoughi R 2003 Microwave reflection and dielectric properties of mortar subjected to compression force and cyclically exposed to water and sodium chloride solution IEEE Trans. Instrum. Meas. $\mathbf{5 2} 111-8$
Pozar D M 2005 Microwave Engineering (New York: Wiley)

Roqueta G, Romeu J and Jofre L 2009 Electromagnetic modeling and characterization of steel fiber reinforced concrete during the pouring process Antennas and Propagation Society Int. Symp. APSURSI '09 (IEEE) pp 1-4

Seagar A D, Barber D C and Brown B H 1987 Theoretical limits to sensitivity and resolution in impedance imaging Clin. Phys. Physiol. Meas. 8 (Suppl A) 13-31

Simons R N 2001 Coplanar Waveguide Circuits, Components and Systems (New York: Wiley-Interscience)

Sokoll T and Jacob A F 2007 In-situ moisture detection system with a vector network analyser Meas. Sci. Technol. 181088

Stuchly S S and Bassey C E 1998 Microwave coplanar sensors for dielectric measurements Meas. Sci. Technol. 91324

Torrents J M, Mason T O, Peled A, Shah S P and Garboczi E J 2001 Analysis of the impedance spectra of short conductive fiber-reinforced composites J. Mater. Sci. 36 4003-12

Volgyi F 2007 Application of microwave aquametry in civil engineering and in power generation Meas. Sci. Technol. 181094

von Hippel A R (ed) 1954 Dielectric Materials and Applications (Cambridge, MA: MIT Press)

Wen C P 1969 Coplanar waveguide: a surface strip transmission line suitable for nonreciprocal gyromagnetic device applications IEEE Trans. Microw. Theory Techn. 17 1087-90

Xu Q, Shi L and Gao C 2002 Measurement of the electromagnetic constants of concrete materials by time-domain reflectometry 3rd Int. Symp. on Electromagnetic Compatibility pp 230-3

Yoo Jin K, Jofre L, De Flaviis F and Feng M Q 2003 Microwave reflection tomographic array for damage detection of civil structures IEEE Trans. Antennas Propag. 51 3022-32 